\title{
The High Prevalence of Diabetes in a Large Cohort of Patients Drawn From Safety Net Clinics
}

\author{
Gregory A. Nichols, PhD; MaryAnn McBurnie, PhD; Ludmilla Paul, MPH; \\ Jennifer E. Potter, MD; Sheila McCann, MS, MEd; Kenneth Mayer, MD; \\ Gerardo Melgar, MD; Sele D’Amato; Jennifer E. DeVoe, MD, DrPhil
}

Suggested citation for this article: Nichols GA, McBurnie M, Paul L, Potter JE, McCann S, Mayer K, et al. The High Prevalence of Diabetes in a Large Cohort of Patients Drawn From Safety Net Clinics. Prev Chronic Dis 2016;13:160056. DOI: http:/ /dx.doi.org/10.5888/pcd13.160056.

\section{PEER REVIEWED}

\section{Abstract}

\section{Introduction}

Underserved populations have been overlooked or underrepresented in research based on data from diabetes registries. We estimated diabetes prevalence using a cohort developed from the electronic health records of 3 networks of safety net clinics that provide care to underserved populations.

\section{Methods}

ADVANCE (Accelerating Data Value Across a National Community Health Center Network) is a partnership of the OCHIN Community Health Information Network (OCHIN), the Health Choice Network (HCN), and the Fenway Health Institute (FHI), representing 97 federally qualified health centers (FQHCs) and 744 clinic sites in 22 US states. Among 952,316 adults with a body mass index (BMI) measurement and at least 2 outpatient visits in 2012 to 2014, we calculated diabetes prevalence using outpatient diagnoses, diagnostic laboratory results, or dispenses of antihyperglycemic agents no more than 730 days apart. We calculated prevalence by age, sex, race, Hispanic ethnicity, and BMI class.

\section{Results}

The crude prevalence of diabetes was $14.4 \%$. Men had a higher prevalence than women ( $16.5 \%$ vs $13.2 \%)$; diabetes prevalence increased across age categories. White patients had the lowest pre- valence (11.4\%) and Hawaiian/Pacific Islanders, the highest prevalence $(21.9 \%$ ), with prevalence ranging from $15.2 \%$ to $16.5 \%$ for other race/ethnicities. The association between BMI class and diabetes prevalence was similar across all racial/ethnic groups.

\section{Conclusion}

The ADVANCE diabetes cohort offers an opportunity to conduct epidemiologic and comparative effectiveness research on underserved and underrepresented individuals, who have a higher prevalence of diabetes than the general US population.

\section{Introduction}

Diabetes is a chronic condition for which multiple office visits, laboratory tests, and pharmacotherapies are recommended on a more than annual basis. As such, the care of patients with diabetes generates a great deal of data that, when available electronically, allows for the creation of registries and the evaluation of trends in diabetes prevalence and clinical management. Indeed, many examples exist, including longstanding health system-specific registries in both the private and public sectors in the United States and national registries in Europe (1-8). Although these and other diabetes registries were originally derived from administrative data, the widespread adoption of electronic health records (EHRs) has allowed for improved accuracy of diabetes identification as well as the creation of networked registries that span multiple health systems (9-11). Recent research has demonstrated the value of identification methods that include comprehensive EHR data (12).

To our knowledge, comprehensive diabetes registries to date have been created only of insured populations. A diabetes registry of patients at safety net clinics would comprise individuals who are underinsured or uninsured or otherwise vulnerable because of economic status, sexual orientation or gender-identity minority status, low literacy, functional or developmental limitations, or other bar- 
riers to accessing health care. These subgroups are typically omitted from or underrepresented in registries created only from medical records of insured individuals, because EHR data from safety net clinics have, until recently, been unavailable. Furthermore, most of the aforementioned subgroups are known to be at elevated risk of developing diabetes. Our objective was to create and describe a diabetes registry from the EHRs of 3 networks of safety net clinics and to calculate and compare diabetes prevalence by age, sex, racial/ethnicity, and body mass index (BMI) class. The result is a valuable resource for studying diabetes in these populations as well as filling critical gaps in knowledge that result from the absence of underinsured and uninsured individuals in registrybased studies.

\section{Methods}

ADVANCE (Accelerating Data Value Across a National Community Health Center Network) is a partnership of the Community Health Information Network (OCHIN), the Health Choice Network (HCN), and the Fenway Health Institute (FHI). Specifics of ADVANCE and its components have been described elsewhere (13). Briefly, these 3 organizations together represent 97 federally qualified health centers (FQHCs) and 744 clinics in 22 US states, currently providing care to over 1.6 million people. ADVANCE was one of the first 11 clinical data research networks (CDRNs) funded by the Patient-Centered Outcomes Research Institute. Each CDRN was required to create a "weight cohort" of patients within their networks and a "common disease cohort" of their own choosing; ADVANCE chose diabetes as its common disease. The Western Institutional Review Board reviewed and approved the study.

To be included in the ADVANCE weight cohort, adult patients aged 20 or older were required to have at least one outpatient visit to an OCHIN, HCN, or FHI clinic in 2012, 2013, or 2014 with a body weight measured in that 3 -year period and a height measured at any time in 2014 or earlier $(n=1,101,640)$. We used the earliest available weight in the period to calculate body mass index (BMI). We created the ADVANCE diabetes cohort as a subset of the weight cohort using the method described by SUPREME-DM (SUrveillance, PREvention and ManagEment of Diabetes Mellitus) study, except that we were unable to include inpatient diagnoses as an identification criterion (14). This method requires the presence of any combination of 2 "events" from outpatient diagnoses (ICD-9 code 250.x), diagnostic laboratory results (HbAlc $>6.4 \%$, fasting glucose $>125 \mathrm{mg} / \mathrm{dL}$, random glucose $>199 \mathrm{mg} / \mathrm{dL}$ ), or dispensation of anti-hyperglycemic agents (primarily metformin, sulfonylureas, or insulin) no more than 730 days apart. This method does not differentiate between type 1 and type 2 diabetes but captures patients with either type. To be considered for inclusion in the diabetes cohort, we restricted the weight cohort to people with at least 2 outpatient visits in the study period $(n=952,316)$. In addition to BMI, we extracted age, sex, race, and Hispanic ethnicity from the enrollment records of the participating health systems.

We calculated prevalence of diabetes by using adults with qualifying visits and BMI measurements as the denominator and people meeting diabetes criteria as the numerator. Because data on race/ ethnicity are missing for $2.5 \%$ of the sample, we estimated prevalence for racial categories and Hispanic ethnicity only for those for whom these data were available. $P$ values for all comparisons here were statistically significant because of our large sample size.

\section{Results}

Of the 952,316 patients in the ADVANCE weight cohort, 559,134 (58.7\%) were from OCHIN clinics, 373,555 (39.2\%) were from HCN clinics, and 19,627 (2.1\%) were from FHI (Table 1). Age and sex were similar for OCHIN and HCN patients. Because FHI provides care to the LGBT community (in addition to the broader population), these patients were much younger than patients from the other clinics and more likely to be men. About $58 \%$ of OCHIN patients were white, compared with $30 \%$ of HCN's and $72 \%$ of FHI's. BMI was similar for OCHIN and HCN and noticeably lower for FHI.

There were 137,445 patients with diabetes in the ADVANCE weight cohort (14.4\%), (Table 2). Patients with diabetes were considerably older and more likely to be male and nonwhite than were patients without diabetes. About $62 \%$ of patients with diabetes were obese compared with $36.5 \%$ of patients without diabetes $(P$ $<.001)$. The 4 panels in Figure 1 show the crude prevalence of diabetes for people in various demographic strata. Men had a higher prevalence than women ( $16.5 \%$ vs $13.2 \%$, Panel A), and diabetes prevalence increased across age categories (Panel B) $(P<.001$ for all comparisons). Non-Hispanic white patients had the lowest prevalence (11.4\%) and Native Hawaiian or Other Pacific Islanders had the highest prevalence (21.9\%). Diabetes prevalence of patients from other races ranged from $15.2 \%$ to $16.5 \%$ (Panel C) $(P$ $<.001$ for all comparisons). The relationship between BMI class and diabetes prevalence was consistently linear (Panel D) and similar for all racial/ethnic groups (Figure 2).

\footnotetext{
The opinions expressed by authors contributing to this journal do not necessarily reflect the opinions of the U.S. Department of Health and Human Services, the Public Health Service, the Centers for Disease Control and Prevention, or the authors' affiliated institutions.
} 

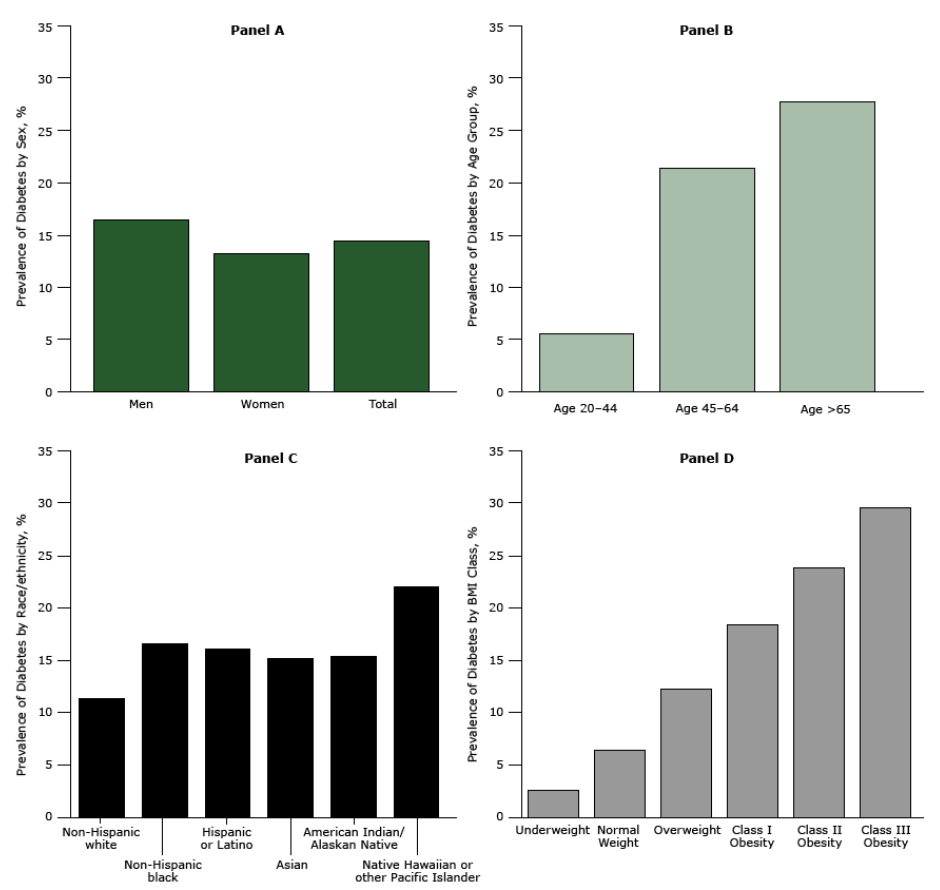

Figure 1. Prevalence of diabetes among ADVANCE study patients by sex and in total (Panel A); age group (Panel B); race/ethnicity (Panel C); and BMI class (Panel D). Body mass index was calculated as measured weight in kilograms divided by height in meters squared.

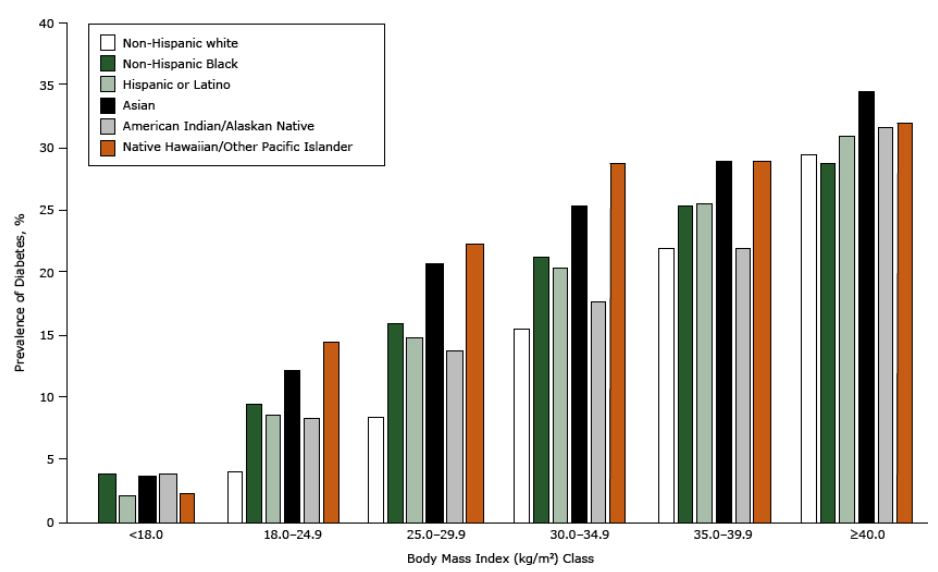

Figure 2. Prevalence of diabetes by body mass index class for selected races and Hispanic ethnicity. Body mass index was calculated as measured weight in kilograms divided by height in meters squared.

\section{Discussion}

The ADVANCE CDRN assembled a cohort of 137,445 adults with diabetes who receive medical care from safety net clinics loc- ated in 22 states ( 6 western states, 2 southwestern states, 2 Midwest states, 4 states in the Great Lakes region, 4 southern states, 2 mid-Atlantic states, Alaska and Hawaii), providing a unique nationwide diabetes registry for conducting patient-centered outcome research among populations that are generally underrepresented in clinical trials and registry-based studies $(11,13)$. In particular, the ADVANCE diabetes cohort allows for the study of diabetes and its complications among uninsured and vulnerable individuals, about whom relatively little is known.

Overall, the crude prevalence of diabetes in the ADVANCE cohort was $14.4 \%$, somewhat higher than the $12.3 \%$ reported in the general US adult population (15). This finding can be partially explained by the larger proportion of high-risk subgroups in ADVANCE. For example, although the ADVANCE diabetes prevalence rates were similar to US rates for Hispanics $(16.1 \%$ vs $17.0 \%$ ) and non-Hispanic blacks (16.5\% vs $14.7 \%)$, the proportions of the ADVANCE population that are Hispanic (30.1\%) or non-Hispanic black (16.5\%) are higher than their proportions in the US population. Although US diabetes prevalence may have increased since 2005 to 2006, crude and age-adjusted rates of diagnosed diabetes have leveled off since 2008 (16). Diabetes prevalence among Asians was only slightly lower than among blacks or Hispanics. Though counterintuitive, this finding is consistent with a report of a similar finding when Asians are aggregated but also demonstrated wide variation of diabetes prevalence among Asians when disaggregated into Chinese, Japanese, Filipino, and South Asian subgroups (17). We cannot provide that level of detail.

Diabetes prevalence and obesity are inextricably entwined, with clear evidence that obesity and weight gain increases the risk of developing diabetes $(18,19)$. Higher diabetes prevalence in the ADVANCE cohort is likely due in part to the cohort's having obesity rates that are substantially higher $(40.0 \%$ vs $32.2 \%)$ than in the general US adult population (20). In our data, the relationship between obesity class and diabetes prevalence was remarkably linear and consistent across all racial/ethnic groups. Thus, despite the known increased risk of diabetes associated with minority groups, the impact of obesity on diabetes risk appears to be uniform. In addition to the health and economic burden of obesity, the risk of mortality increases among the obese despite improving trends in cardiovascular risk factor control $(21,22)$. Recent analyses suggest that the previously increasing trends in obesity are leveling off in the general US population $(20,23)$; it is unknown whether a similar plateau is also occurring in safety net populations. Nevertheless, despite a flattening of obesity trends, there are now more overweight and obese young people than ever before (20). Because earlier onset of obesity increases the risk of diabetes, we may witness a second wave of the diabetes epidemic in the near future (24). 
Insurance status may also play a role in our study's higher prevalence rates of diabetes. Although directly comparable data are elusive, about $16 \%$ of the US adult population did not have health insurance in 2012, whereas approximately $25 \%$ of the ADVANCE cohort was uninsured (25). Uninsured diabetes patients are less likely to receive preventive care or to know about their diabetes, and more likely to have poor glycemic control (26-28). Studies of the long-term ramifications of these disparities are needed and require datasets such as the ADVANCE data warehouse to execute them properly. The Affordable Care Act will provide insurance to many of those currently lacking it. Early indications are that uninsured community health center visits have substantially decreased and that more Medicaid patients with diabetes are receiving a diagnosis and being treated earlier $(25,29)$. The long-term implications of such changes demand careful evaluation. We could not determine insurance status on an individual level, primarily because it can change from one encounter to another.

In addition to the uniqueness of the ADVANCE cohort, strengths include its size and the use of methods to identify diabetes that are consistent with the SUPREME-DM cohort and other electronic database definitions that capture both patients with diagnosed diabetes and patients with undiagnosed diabetes $(12,29)$. Consistency with other cohorts presents the opportunity for collaborative efforts that could create a comprehensive and nationally representative database of all patients with diabetes.

There are also limitations to consider. First, inpatient data are a work-in-progress in ADVANCE and are not available. Although this limits our ability to examine some outcomes, lack of inpatient diagnoses has little impact on the identification of patients with diabetes (12). Second, similar to all diabetes registries and cohorts derived from EHR or administrative data, the ADVANCE cohort comprises clinic attendees who sought health care, thus excluding patients with undiagnosed and untreated disease. Because patients who do not routinely access health services are more likely to be from racial/ethnic minority communities, the diabetes estimates in this safety net $\mathrm{CHC}$ sample may underrepresent the prevalence of the disease in the most disenfranchised populations. Conversely, because the ADVANCE cohort is created from people with clinic contacts, it differs from health system-based cohorts such as SUPREME-DM that can include total enrollment as the denominator. This difference may have artificially increased prevalence estimates in ADVANCE. Furthermore, by requiring patients to have a BMI on record, we may have overselected patients who have diabetes or are at risk for its development. There may also be variation among sites represented in the 3 ADVANCE networks regarding data capture and clinician coding and documentation of diabetes. However, the SUPREME-DM algorithm that we used is a robust method that identifies more patients with diabetes than other electronic phenotype definitions (12). This is due in part to the inclusion of laboratory data that captures patients who may not have received a diagnosis (30). Unfortunately, the algorithm does not differentiate between type 1 and type 2 diabetes, and differences by diabetes type may exist. Finally, we believe that insurance status may have played a role in our results but cannot reliably differentiate between uninsured, underinsured, and fully insured patients on an individual level.

Despite these limitations, the ADVANCE diabetes cohort offers an opportunity to conduct epidemiologic and comparative effectiveness research in underserved and underrepresented individuals. This resource provides the ability to quickly identify safety net patients for randomized studies, to examine longitudinal trends, and to assess care practices and identify potential disparities within and across registries. As part of the larger PCORnet (National Patient-Centered Clinical Research Network) that is using identical methods across other CDRNs, and by using methods similar to SUPREME-DM, enormous opportunity exists for collaborations that could generate a more representative national database of people with diabetes, thus providing results that are more generalizable to the US population.

\section{Acknowledgments}

This study was funded by Patient-Centered Outcomes Research Institute award no. CDRN-1306-04716.

\section{Author Information}

Corresponding Author: Gregory A. Nichols, PhD, Center for Health Research, 3800 N Interstate Ave, Portland, OR 972271098. Telephone: 503-335-6733. Email: greg.nichols@kpchr.org.

Author Affiliations: MaryAnn McBurnie, Kaiser Permanente Center for Health Research, Portland, Oregon; Ludmilla Paul, Sheila McCann, Health Choice Network of Florida, Miami, Florida; Jennifer E. Potter, Beth Israel Deaconess Medical Center, Boston, Massachusetts; Kenneth Mayer, Beth Israel Deaconess Medical Center, and The Fenway Institute, Boston, Massachusetts; Gerardo Melgar, Cowlitz County Family Health Center, Longview, Washington; Sele D'Amato, OCHIN Patient Engagement Panel, Portland, Oregon; Jennifer E. DeVoe, Oregon Health and Science University, and OCHIN, Portland, Oregon.

\section{References}

1. Brown JB, Nichols GA, Glauber HS. Case-control study of 10 years of comprehensive diabetes care. West J Med 2000; 172(2):85-90.

\footnotetext{
The opinions expressed by authors contributing to this journal do not necessarily reflect the opinions of the U.S. Department of Health and Human Services, the Public Health Service, the Centers for Disease Control and Prevention, or the authors' affiliated institutions.
} 
2. Selby JV. Linking automated databases for research in managed care settings. Ann Intern Med 1997;127(8 Pt 2):719-24.

3. O’Connor PJ, Rush WA, Pronk NP, Cherney LM. Identifying diabetes mellitus or heart disease among health maintenance organization members: sensitivity, specificity, predictive value, and cost of survey and database methods. Am J Manag Care 1998;4(3):335-42.

4. Newton KM, Wagner EH, Ramsey SD, McCulloch D, Evans $\mathrm{R}$, Sandhu N, et al. The use of automated data to identify complications and comorbidities of diabetes: a validation study. J Clin Epidemiol 1999;52(3):199-207.

5. Wilson C, Susan L, Lynch A, Saria R, Peterson D. Patients with diagnosed diabetes mellitus can be accurately identified in an Indian Health Service patient registration database. Public Health Rep 2001;116(1):45-50.

6. Miller DR, Safford MM, Pogach LM. Who has diabetes? Best estimates of diabetes prevalence in the Department of Veterans Affairs based on computerized patient data. Diabetes Care 2004;27(Suppl 2):B10-21.

7. Green A, Sortsø C, Jensen PB, Emneus M. Validation of the danish national diabetes register. Clin Epidemiol 2014;7:5-15.

8. Gudbjörnsdottir S, Cederholm J, Nilsson PM, Eliasson B; Steering Committee of the Swedish National Diabetes Register. The National Diabetes Register in Sweden: an implementation of the St. Vincent Declaration for Quality Improvement in Diabetes Care. Diabetes Care 2003; 26(4):1270-6.

9. Tang PC, Ralston M, Arrigotti MF, Qureshi L, Graham J. Comparison of methodologies for calculating quality measures based on administrative data versus clinical data from an electronic health record system: implications for performance measures. J Am Med Inform Assoc 2007;14(1):10-5.

10. Kudyakov R, Bowen J, Ewen E, West SL, Daoud Y, Fleming $\mathrm{N}$, et al. Electronic health record use to classify patients with newly diagnosed versus preexisting type 2 diabetes: infrastructure for comparative effectiveness research and population health management. Popul Health Manag 2012; 15(1):3-11.

11. Nichols GA, Desai J, Elston Lafata J, Lawrence JM, O’Connor PJ, Pathak RD, et al.;SUPREME-DM Study Group. Construction of a multisite DataLink using electronic health records for the identification, surveillance, prevention, and management of diabetes mellitus: the SUPREME-DM project. Prev Chronic Dis 2012;9:E110.

12. Richesson RL, Rusincovitch SA, Wixted D, Batch BC, Feinglos MN, Miranda ML, et al. A comparison of phenotype definitions for diabetes mellitus. J Am Med Inform Assoc 2013;20(e2):e319-26.
13. DeVoe JE, Gold R, Cottrell E, Bauer V, Brickman A, Puro J, et al. The ADVANCE network: accelerating data value across a national community health center network. J Am Med Inform Assoc 2014;21(4):591-5.

14. Nichols GA, Schroeder EB, Karter AJ, Gregg EW, Desai J, Lawrence JM, et al.;SUPREME-DM Study Group. Trends in diabetes incidence among 7 million insured adults, 2006-2011: the SUPREME-DM project. Am J Epidemiol 2015; 181(1):32-9.

15. Centers for Disease Control and Prevention. National Diabetes Statistics Report: estimates of diabetes and its burden in the United States, 2014. Atlanta (GA): US Department of Health and Human Services; 2014.

16. Centers for Disease Control and Prevention. Crude and ageadjusted rates of diagnosed diabetes per 100 civilian, noninstitutionalized adult population, United States, 1980-2014. http://www.cdc.gov/diabetes/statistics/prev/national/ figageadult.htm. Accessed August 24, 2015.

17. Karter AJ, Schillinger D, Adams AS, Moffet HH, Liu J, Adler $\mathrm{NE}$, et al. Elevated rates of diabetes in Pacific Islanders and Asian subgroups: The Diabetes Study of Northern California (DISTANCE). Diabetes Care 2013;36(3):574-9.

18. Maggio CA, Pi-Sunyer FX. Obesity and type 2 diabetes. Endocrinol Metab Clin North Am 2003;32(4):805-22, viii.

19. Biggs ML, Mukamal KJ, Luchsinger JA, Ix JH, Carnethon MR, Newman AB, et al. Association between adiposity in midlife and older age and risk of diabetes in older adults. JAMA 2010;303(24):2504-12.

20. Ogden CL, Carroll MD, Kit BK, Flegal KM. Prevalence of childhood and adult obesity in the United States, 2011-2012. JAMA 2014;311(8):806-14.

21. Wang YC, McPherson K, Marsh T, Gortmaker SL, Brown M. Health and economic burden of the projected obesity trends in the USA and the UK. Lancet 2011;378(9793):815-25.

22. Gregg EW, Cheng YJ, Cadwell BL, Imperatore G, Williams DE, Flegal KM, et al. Secular trends in cardiovascular disease risk factors according to body mass index in US adults. JAMA 2005;293(15):1868-74.

23. Flegal KM, Carroll MD, Kit BK, Ogden CL. Prevalence of obesity and trends in the distribution of body mass index among US adults, 1999-2010. JAMA 2012;307(5):491-7.

24. The NS, Richardson AS, Gordon-Larsen P. Timing and duration of obesity in relation to diabetes: findings from an ethnically diverse, nationally representative sample. Diabetes Care 2013;36(4):865-72.

25. Angier H, Hoopes M, Gold R, Bailey SR, Cottrell EK, Heintzman J, et al. An early look at rates of uninsured safety net clinic visits after the Affordable Care Act. Ann Fam Med 2015;13(1):10-6.

The opinions expressed by authors contributing to this journal do not necessarily reflect the opinions of the U.S. Department of Health and Human Services, the Public Health Service, the Centers for Disease Control and Prevention, or the authors' affiliated institutions. 
26. Gold R, DeVoe JE, McIntire PJ, Puro JE, Chauvie SL, Shah AR. Receipt of diabetes preventive care among safety net patients associated with differing levels of insurance coverage. J Am Board Fam Med 2012;25(1):42-9.

27. Decker SL, Kostova D, Kenney GM, Long SK. Health status, risk factors, and medical conditions among persons enrolled in Medicaid vs uninsured low-income adults potentially eligible for Medicaid under the Affordable Care Act. JAMA 2013; 309(24):2579-86.

28. Ali MK, McKeever Bullard K, Imperatore G, Barker L, Gregg EW;Centers for Disease Control and Prevention (CDC). Characteristics associated with poor glycemic control among adults with self-reported diagnosed diabetes - National Health and Nutrition Examination Survey, United States, 2007-2010. MMWR Suppl 2012;61(2):32-7.

29. Kaufman HW, Chen Z, Fonseca VA, McPhaul MJ. Surge in Medicaid patients in 2014 within Medicaid expansion states under the Affordable Care Act. Diabetes Care 2015; 38(5):833-7.

30. Zgibor JC, Orchard TJ, Saul M, Piatt G, Ruppert K, Stewart A, et al. Developing and validating a diabetes database in a large health system. Diabetes Res Clin Pract 2007;75(3):313-9.

The opinions expressed by authors contributing to this journal do not necessarily reflect the opinions of the U.S. Department of Health and Human Services, the Public Health Service, the Centers for Disease Control and Prevention, or the authors' affiliated institutions. 


\section{Tables}

Table 1. Characteristics of the ADVANCE Population Used to Identify Diabetes, by Participating Health System, 2012-2014

\begin{tabular}{|c|c|c|c|c|}
\hline Characteristics & FHI & $\mathrm{HCN}$ & OCHIN & Total \\
\hline Total patients, $n$ & 19,627 & 373,555 & 559,134 & 952,316 \\
\hline Mean age, y (SD) & $39.1(13.5)$ & $45.7(15.4)$ & $46.8(16.5)$ & $46.2(16.1)$ \\
\hline \multicolumn{5}{|l|}{ Age group, $y$} \\
\hline $20-44$ & $67.2 \%$ & $48.4 \%$ & $48.0 \%$ & $48.5 \%$ \\
\hline $45-64$ & $28.8 \%$ & $41.6 \%$ & $37.7 \%$ & $39.0 \%$ \\
\hline$\geq 65$ & $4.1 \%$ & $10.1 \%$ & $14.3 \%$ & $12.5 \%$ \\
\hline \multicolumn{5}{|l|}{ Sex } \\
\hline Men & $62.9 \%$ & $34.1 \%$ & $39.3 \%$ & $37.7 \%$ \\
\hline Women & $37.1 \%$ & $65.9 \%$ & $60.7 \%$ & $62.3 \%$ \\
\hline \multicolumn{5}{|l|}{ Race/ethnicity } \\
\hline American Indian/Alaska Native & $0.2 \%$ & $0.2 \%$ & $0.6 \%$ & $0.4 \%$ \\
\hline Asian & $6.0 \%$ & $1.8 \%$ & $3.0 \%$ & $2.6 \%$ \\
\hline Non-Hispanic black & $7.3 \%$ & $22.2 \%$ & $12.9 \%$ & $16.5 \%$ \\
\hline Hispanic or Latino & $9.5 \%$ & $43.1 \%$ & $22.1 \%$ & $30.1 \%$ \\
\hline Multiple race & $1.8 \%$ & $0.7 \%$ & $0.5 \%$ & $0.6 \%$ \\
\hline Native Hawaiian/Other Pacific Islander & $0.5 \%$ & $0.4 \%$ & $0.3 \%$ & $0.4 \%$ \\
\hline Unknown & $1.5 \%$ & $2.0 \%$ & $2.9 \%$ & $2.5 \%$ \\
\hline Non-Hispanic white & $71.9 \%$ & $29.6 \%$ & $57.7 \%$ & $47.0 \%$ \\
\hline Other & $1.4 \%$ & $0.0 \%$ & $0.1 \%$ & $0.1 \%$ \\
\hline Mean body mass index, $\mathrm{kg} / \mathrm{m}^{2}(\mathrm{SD})$ & $26.8(5.6)$ & $29.9(7.3)$ & $29.6(7.4)$ & $29.7(7.4)$ \\
\hline \multicolumn{5}{|l|}{ BMI class } \\
\hline Underweight $\left(<18.5 \mathrm{~kg} / \mathrm{m}^{2}\right)$ & $3.4 \%$ & $3.8 \%$ & $3.4 \%$ & $3.6 \%$ \\
\hline Normal weight (18.5-24.9 kg/m²) & $41.0 \%$ & $23.5 \%$ & $26.0 \%$ & $25.3 \%$ \\
\hline Overweight $(25-29.9$ kg/m²) & $33.5 \%$ & $31.3 \%$ & $30.7 \%$ & $31.0 \%$ \\
\hline Class I obesity $\left(30-34.9 \mathrm{~kg} / \mathrm{m}^{2}\right)$ & $13.7 \%$ & $21.8 \%$ & $20.6 \%$ & $20.9 \%$ \\
\hline Class II obesity $(35-39.9$ kg/m²) & $5.1 \%$ & $10.7 \%$ & $10.4 \%$ & $10.4 \%$ \\
\hline Class III obesity $\left(\geq 40 \mathrm{~kg} / \mathrm{m}^{2}\right)$ & $3.3 \%$ & $8.8 \%$ & $8.8 \%$ & $8.7 \%$ \\
\hline
\end{tabular}

Abbreviations: ADVANCE, Accelerating Data Value Across a National Community Health Center Network; OCHIN, Community Health Information Network; HCN, Health Choice Network; FHI, Fenway Health Institute; SD, standard deviation; kg/ $\mathrm{m}^{2}$, kilograms divided by height in meters squared. 
Table 2. ADVANCE Patients by Diabetes Status, 2012-2014

\begin{tabular}{|c|c|c|}
\hline Patient Characteristics & No Diabetes & Diabetes \\
\hline Total patients, $\mathrm{N}$ & 814,871 & 137,445 \\
\hline Percentage of total & $85.6 \%$ & $14.4 \%$ \\
\hline \multicolumn{3}{|l|}{ Age group, $y$} \\
\hline $20-44$ & $53.6 \%$ & $18.3 \%$ \\
\hline $45-64$ & $35.9 \%$ & $57.8 \%$ \\
\hline$\geq 65$ & $10.5 \%$ & $23.9 \%$ \\
\hline \multicolumn{3}{|l|}{ Sex } \\
\hline Men & $36.8 \%$ & $43.2 \%$ \\
\hline Women & $63.2 \%$ & $56.8 \%$ \\
\hline \multicolumn{3}{|l|}{ Race/ethnicity } \\
\hline American Indian/Alaska Native & $0.4 \%$ & $0.5 \%$ \\
\hline Asian & $2.5 \%$ & $3.0 \%$ \\
\hline Non-Hispanic black & $15.7 \%$ & $20.7 \%$ \\
\hline Hispanic or Latino & $29.2 \%$ & $35.1 \%$ \\
\hline Multiple race & $0.6 \%$ & $0.5 \%$ \\
\hline Native Hawaiian/other Pacific Islander & $0.3 \%$ & $0.6 \%$ \\
\hline Unknown & $2.7 \%$ & $1.7 \%$ \\
\hline Non-Hispanic white & $48.5 \%$ & $37.9 \%$ \\
\hline Other & $0.1 \%$ & $0.0 \%$ \\
\hline \multicolumn{3}{|l|}{ BMI class, kg/m² } \\
\hline Underweight $(<18.5)$ & $4.1 \%$ & $0.7 \%$ \\
\hline Normal weight (18.5-24.9) & $27.7 \%$ & $11.3 \%$ \\
\hline Overweight (25- $\geq 29.9$ ) & $31.8 \%$ & $26.4 \%$ \\
\hline Class I obesity (30-34.9) & $20.0 \%$ & $26.7 \%$ \\
\hline Class II obesity (35-39.9) & $9.3 \%$ & $17.2 \%$ \\
\hline Class III obesity $(\geq 40)$ & $7.2 \%$ & $17.8 \%$ \\
\hline
\end{tabular}

Abbreviation: ADVANCE, Accelerating Data Value Across a National Community Health Center Network. 